\title{
Concept of Paradigm Shift in Public Health Research
}

\author{
Khanal K \\ Department of Community Medicine, \\ Kathmandu University School of Medical Sciences, Dhulikhel, Nepal.
}

Research work varies between researchers as they have different beliefs and ways of viewing and interacting within their environment. However, there are certain standards and rules that guide a researcher's beliefs and actions. Such standards or principles can be referred to as a paradigm. The paradigm guides researchers to understand and choose a particular methodology with an understanding of the reasons behind their approach. ${ }^{1}$ A paradigm consists of four elements; namely body of theory for the research, the background metaphysical assumptions behind the theory, the epistemological values including norms of rationality of the theory and finally it's exemplars. ${ }^{2}$

Paradigms are shifted by change of one theory to another in course of time leading to revolution of scientific thinking over a period of time. The following stages of paradigm has been suggested in a development of science. ${ }^{2}$ The first stage is called "Pre-Paradigm". In this stage, people seeking to understand an observed phenomenon share no common background theory. There is confusion as multiple paradigms are put forward by different schools of thought and scientists may disagree with one another as they propose and support their individual theories. Eventually, one paradigm wins through and becomes the dominant principle. The second stage is "Normal Science" where the scientists normally accepts the core elements of the paradigm as a dogma. They perform experiments that test and prove its efficacy in a range of situation. During normal science, a paradigm gets worked out in detail. In the course of normal science problems that refuse to accept resolution with the paradigm consistently arise. If these "recalcitrant" remain problems long enough, they are considered as "anomalies." When the anomalies become harder and harder to ignore the reseachers focus and scrutinize the anomalies and attempt to resolve anomalies that give arise to a crisis in normal science. Such a crisis creats question on the core elements of a paradigm that had been previously held dogmatically. In this situation, it is possible for scientists to propose and win wide accepance for significant changes in the theoritical framework. This is called "scientific resolution" in which the scientific community dumps one paradigm in favour of another. When new paradigm takes hold, normal science is resumed and continuos to work until new batch of anomolies occurs.

There are basically two types of research paradigm which are used widely by public health reseachers to solve the health related issues. ${ }^{3}$ First, Positivist Paradigm : This paradigm is closely associated with quantitative research approach. In this approach, it is assumed that there is fixed orderly reality that can be objectively studied and tested by hard statistical tests. This paradigm emerged as a philosophical paradigm in the 19th century with Auguste Comte's rejection of metaphysics and his assertion that only scientific knowledge can reveal the truth about reality. ${ }^{4}$ This is known as early positivism and also known as reductionism where sociology is reduced to different sciences and mathematics. This was established as the dominant scientific method in the early part of the 20th century by members of the Vienna Circle. ${ }^{4}$ The Vienna Circle put demarcation criteria for what is science and what is not. Defined science can be verified to be objectively true by this method. However, such varification in a public health scinario by researchers is a daunting task and is likely to bring more questions than answers. ${ }^{4}$ Second, Naturalistic Paradigm : In this paradigm, the reseacher assumes that there are multiple interpretations of realiality and people construct their own reality based on their socioeconomic context. This is totally subjective approach which is valid for interpretation of respondents feeling. However, the findings depend on the interaction between the respondents and the researcher. This approach helps to develop a theory/framework to explain the issue under observation. ${ }^{3}$

Public health research has gone through paradigm shifts over the years. ${ }^{5}$ Prior to the 1990 s, the global policy discussed that the population growth can be reduced through controlling women's fertility and promotes family planning for good health of women. The different women organization and its allies around the world succeeded in raising awareness of the importance of the broader health and social context of fertility and parenthood, sexuality, gender and human rights. These shifts culminated in a series of United Nations conferences from 1990 to 1995(Vienna human rights conference 1993, Cairo international conference in 
population and development 1994 and the fourth world conference on Women in Beijing,1995) and endorsed consensus documents that recognized the complex links among population growth, sexual and reproductive health, gender, equality, consumption and production patterns, sustainable development and human rights. The international agreement highlights that sexual and reproductive health and rights are related to women's rights and well-being which are key to social and economic development. ${ }^{5}$

Another example of paradigm shift in public health is childhood nutrition. Approaches to childhood malnutrition in public health in under resourced countries have changed since Second World War. Under nutrition in children was thought to be due purely to lack of appropriate food of different categories leading to known clinical manifestations. It is now clear that it is not just the availability of food but other factors like knowledge about the food, eating habits and co-exiting morbidity also determines the clinical manifestation of under nutrition of different categories. These shifts in paradigm are due to new scientific discoveries, changing interpretations of science, and competing ethical priorities and positions. A sequence of paradigm shift in public health nutrition is like this: protein deficiency paradigm (1950 to 1974), multi-sectoral nutrition planning and national nutrition policies paradigm (1973 to 1985), community-based nutrition and primary health care paradigm (1985 to 1995), micronutrient paradigm (1995 to 2005). Today, the competing two paradigms on public health nutrition are on investment in nutrition, and the human rights approach with malnourished children as their focus. ${ }^{6}$

Thus, it is very important to understand that paradigms are important parts of research and philosophy which guide us how we make decisions and carry out research activities in public health science.

\section{REFERENCES}

1. The University of Notre Dame. Methodology [Internet]. Accesed on 17 Dec 2012. Available from: http://researchonline.nd.edu.au/cgi/ viewcontent.cgi?filename $=2 \&$ article $=1030 \&$ context $=$ theses $\&$ type $=$ additional

2. Payne WR. Thomas Kuhn [Internet]. Belleveu College. Available from: http://personal.bellevuecollege.edu/wpayne/thomas_kuhn. htm

3. Joshi SP. Qualitative Research: Approaches for health personnel. 1st ed. Kathmandu: Makalu Publication House; 2008.

4. Bärmark J, Thorpenberg S. Theory of Science [Unpublished document]. University of Gothenburg, Sweden. Accessed on 2012 November 26.

5. UNFPA. Sexual and Reproductive Health for All: Reducing poverty, advancing development and protecting human rights. New York: UNFPA;2010.

6. Jonsson U. The rise and fall of paradigms in world food and nutrition policy. Journal of the World Public Health Nutrition Association 2010;1(3). [Internet]. Assesed on 3rd Mar 2013 Available from: http://www.wphna.org/wn_commentaryUJ_July2010.asp 